\title{
1 Preimmunization correlates of protection 2 shared across malaria vaccine trials in adults
}

3

4 Maxwell L. Neal ${ }^{1}$, Fergal J. Duffy ${ }^{1}$, Ying Du ${ }^{1}$, John D. Aitchison ${ }^{1,2,3^{*}}$, Kenneth D. Stuart ${ }^{1,4,5^{*}}$

$5{ }^{1}$ Center for Global Infectious Disease Research, Seattle Children's Research Institute, Seattle, WA, 6 United States

$7 \quad 2$ Department of Pediatrics, University of Washington, Seattle, WA, United States

$8{ }^{3}$ Department of Biochemistry, University of Washington, Seattle, WA, United States

$9{ }^{4}$ Department of Global Health, University of Washington, Seattle, WA, United States

$10{ }^{5}$ Vaccine and Infectious Disease Division, Fred Hutchinson Cancer Research Center, Seattle, WA, United 11 States

* Co-corresponding authors

17 Ken.Stuart@seattlechildrens.org 


\section{Abstract}

19 Identifying preimmunization biological characteristics that promote an effective vaccine

20 response offers opportunities for illuminating the critical immunological mechanisms that confer

21 vaccine-induced protection, for developing adjuvant strategies, and for tailoring vaccination

22 regimens to individuals or groups. In the context of malaria vaccine research, studying

23 preimmunization correlates of protection can help address the need for a widely-effective

24 malaria vaccine, which remains elusive. In this study, common preimmunization correlates of

25 protection were identified using transcriptomic data from four independent, heterogeneous

26 malaria vaccine trials in adults. Systems-based analyses showed that a moderately elevated

27 inflammatory state prior to immunization was associated with protection against malaria

28 challenge. Functional profiling of protection-associated genes revealed the importance of

29 several inflammatory pathways, including TLR signaling. These findings, which echo previous

30 studies that associated enhanced preimmunization inflammation with protection, illuminate

31 common baseline characteristics that set the stage for an effective vaccine response across

32 diverse malaria vaccine strategies in adults. 


\section{Introduction}

Whether a vaccine generates an immune response that results in protection from infection or disease depends in part on the preimmunization status and capacities of the vaccinated individual's immune system ${ }^{1}$. Identifying preimmunization correlates of protection, therefore, offers opportunities for illuminating the immunological pathways that promote or attenuate protective vaccine responses, for identifying avenues whereby the immune system can be influenced to respond effectively, and for developing personalized approaches that predict vaccine efficacy on an individual or group basis. These opportunities can potentially benefit malaria vaccine development efforts: Although substantial progress has been made developing anti-malaria vaccines, a vaccine that is widely-effective has remained elusive ${ }^{2-5}$, and it remains unclear why malaria vaccines induce a protective response in some individuals but not in others. Exploring the preimmunization correlates of protection in malaria vaccine trials presents an opportunity to identify the immunological conditions that set the stage for vaccine-induced responses that are protective against future infection and to help guide the rational design of more effective vaccines. While the list of studies in this research area is not extensive, some preimmunization correlates of protection have been identified for vaccines against influenza ${ }^{6-9}$, hepatitis $\mathrm{B}^{10-12}$, yellow fever ${ }^{13}$ and malaria ${ }^{14-21}$.

To identify common preimmunization correlates of protection across various malaria vaccine trials, we performed transcriptomic analysis of four independent trials: the Immunization via

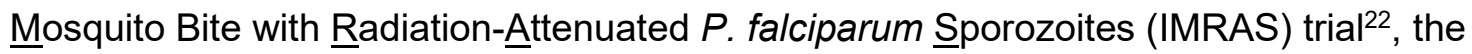
Bagamoyo SPoroZzoite Vaccination 1 (BSPZV1) trial ${ }^{23}$, the MAL68 RTS,S vaccine trial ${ }^{24}$, and a Chloroquine Prophylaxis and Sporozoites (CPS) trial ${ }^{25,26}$. These trials varied in the vaccines administered (live, radiation-attenuated whole sporozoites, RTS,S subunit vaccine or live sporozoites where establishment of blood stage infection was prevented by chloroquine chemoprophylaxis), the route of administration (intravascular injection, intramuscular injection, or mosquito bite) the country of trial subject recruitment (USA, Tanzania, or The Netherlands), whether subjects were malaria-naïve prior to immunization (USA, The Netherlands) or experienced (Tanzania), and the source of transcriptomic material (whole blood or peripheral blood mononuclear cells - PBMCs). Each trial included a challenge phase, wherein subjects were infected with infectious $P$. falciparum to determine whether the subject mounted a vaccine response protective against malaria. To our knowledge, this study is the first to investigate preimmunization correlates of protection from transcriptomics across multiple, heterogenous malaria vaccine trials in adults. Despite the wide-ranging clinical trial conditions, our results indicate that protection against post-immunization malaria challenge is associated with innate immune system activation and a moderately elevated inflammatory state prior to immunization, including higher expression in genes associated with TLR signaling and other inflammatory pathways. These results echo molecular- and pathway-level preimmunization correlates of malaria protection derived from previous analyses on narrower sets of clinical trials ${ }^{19,21}$. They highlight specific immunological pathways that may play critical roles in the development of effective malaria vaccine-elicited responses and offer potential targets for manipulating the immune system into a state that promotes such responses. 


\section{Results}

\section{Correlates of protection among pooled preimmunization}

\section{7 transcriptomes}

78 Preimmunization transcriptomes were pooled from the four malaria vaccine trials and consisted of 84 samples (Table 1, Methods). A differential expression analysis comparing the transcriptomes of all protected participants $(n=38)$ and all non-protected participants $(n=46)$ pooled from the trials revealed no genes with statistically-significant differences in transcript abundance using an FDR-adjusted $P$-value cutoff of 0.1 . To test for differences among functionally-related groups of genes, we performed Gene Set Enrichment Analysis (GSEA ${ }^{27}$ to identify statistically-significant overrepresentation of predefined gene sets including immunological blood transcriptional modules ${ }^{28,29}$ and the MSigDB Hallmark gene sets ${ }^{30}$. GSEA results on genes ranked according to their DESeq2 Wald test statistic revealed significant differences in transcript abundances (FDR-adjusted $P$-value $<0.05$ ) among 207 gene sets. Gene sets enriched for higher abundance transcripts in protected subjects were predominantly associated with inflammation and inflammatory signaling pathways, myeloid lineage cells including monocytes and neutrophils, coagulation, antigen presentation, cell death and apoptosis, the endoplasmic reticulum, the extracellular matrix, and the complement system (Fig. 1). Gene sets enriched for lower abundance transcripts in protected subjects were predominantly associated with the cell cycle, protein synthesis, interferon, T cells, mitochondria stress/respiration, and NK cells. As illustrated in Fig. 2, leading-edge genes (those primarily responsible for the significant enrichment scores due to non-random grouping at the tails of a ranked list) from gene sets with the highest and lowest normalized enrichment scores (NESs) showed moderate differences in transcript abundance between protected and non-protected subjects. For example, the top 10 leading-edge genes in Fig. 2a showed a mean $\log _{2}$ foldchange of $0.41 \pm 0.22$ (SD) between protected and non-protected subjects. Our analysis revealed that, when comparing protected and non-protected subjects, differences in transcript abundance of gene sets showed statistical significance, whereas differences at the individual

102 gene level did not.

Table 1. Characteristics of the four clinical trials that generated transcriptomic data used to identify preimmunization correlates of protection.

\begin{tabular}{|c|c|c|c|c|c|c|c|}
\hline $\begin{array}{c}\text { Trial name } \\
\text { (ClinicalTrials.gov ID) }\end{array}$ & Immunogen & $\begin{array}{l}\text { Recruitment } \\
\text { country }\end{array}$ & $\begin{array}{l}\text { Sample } \\
\text { source }\end{array}$ & $\begin{array}{c}N \\
\text { preimmunization } \\
\text { samples }\end{array}$ & $\begin{array}{c}\text { Prior } \\
\text { malaria } \\
\text { exposure }\end{array}$ & $\begin{array}{c}N \text { female } I \\
\text { male }\end{array}$ & $\begin{array}{l}N \text { challenge- } \\
\text { protected / } \\
\text { non-protected }\end{array}$ \\
\hline $\begin{array}{c}\text { IMRAS } \\
\text { Immunization via Mosquito Bite with } \\
\text { Radiation-Attenuated P. falciparum } \\
\text { Sporozoites } \\
\text { (NCT01994525) }\end{array}$ & $\begin{array}{l}\text { Radiation-attenuated } \\
\text { sporozoites via mosquito bite }\end{array}$ & U.S.A. & Whole blood & 11 & Naïve & $1 / 10$ & $6 / 5$ \\
\hline $\begin{array}{c}\text { BSPZV1 } \\
\text { Bagamoyo } \underline{\text { SPoroZzoite Vaccination } 1} \\
(\text { NCT02132299) }\end{array}$ & $\begin{array}{l}\text { Radiation-attenuated } \\
\text { sporozoites via IV injection }\end{array}$ & Tanzania & Whole blood & 22 & Non-naïve & $0 / 22$ & $8 / 14$ \\
\hline $\begin{array}{c}\text { MAL68 } \\
\text { (NCT01366534) }\end{array}$ & $\begin{array}{l}\text { RTS,S/A01 } \pm \text { Ad35.CS.01 } \\
\text { vaccine via injection }\end{array}$ & U.S.A. & PBMCs & 45 & Naïve & $20 / 25$ & $21 / 24$ \\
\hline 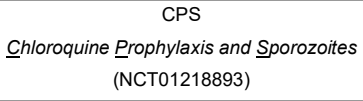 & $\begin{array}{l}\text { Sporozoites via mosquito bite } \\
\text { (under chloroquine cover) }\end{array}$ & $\begin{array}{l}\text { The } \\
\text { Netherlands }\end{array}$ & Whole blood & 7 & Naïve & $3 / 4$ & $3 / 4$ \\
\hline
\end{tabular}


medRxiv preprint doi: https://doi.org/10.1101/2021.08.13.21262031; this version posted September 5, 2021. The copyright holder for this preprint (which was not certified by peer review) is the author/funder, who has granted medRxiv a license to display the preprint in perpetuity.

All rights reserved. No reuse allowed without permission.

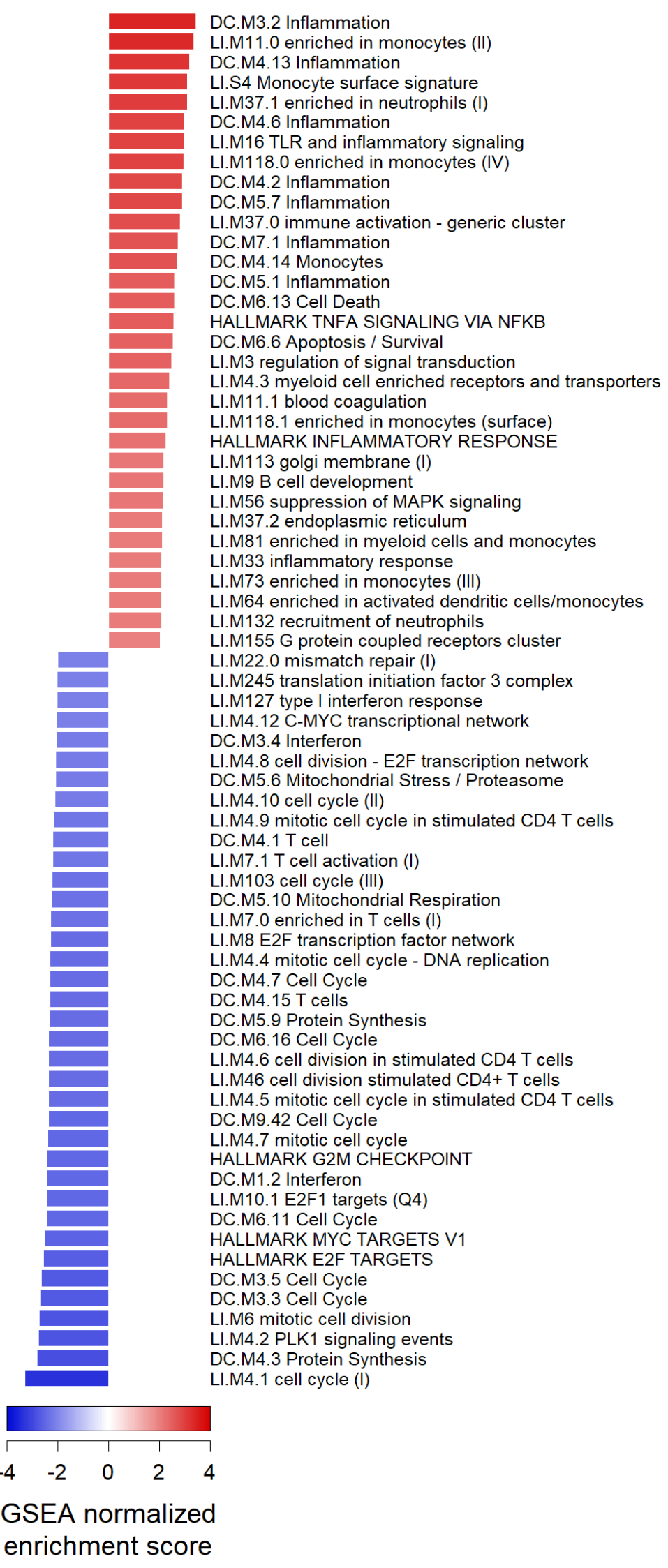

Figure 1. GSEA results on the pooled set of preimmunization transcriptomes from four malaria vaccine trials. Gene sets with positive GSEA normalized enrichment scores indicate they are significantly enriched for genes with higher transcript abundance in protected subjects; negative scores indicate significant enrichment for genes with lower transcript abundance in protected subjects. Gene sets shown have absolute scores $>2.0$ and FDRadjusted $P$-values $<0.05$. Sets named "Undetermined" or "TBA" are not shown. 
a DC.M3.2 Inflammation
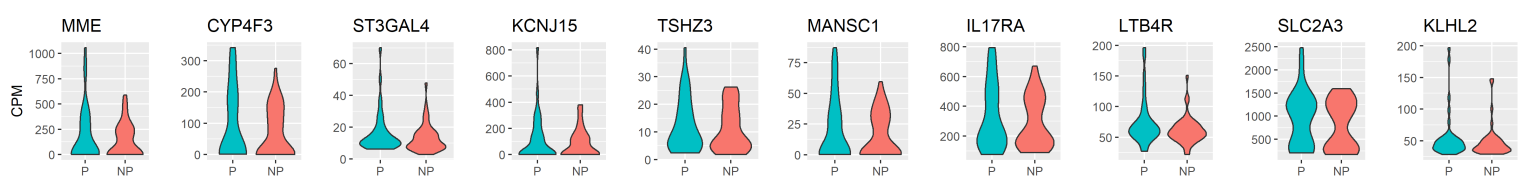

\section{b LI.M4.1 cell cycle (I)}
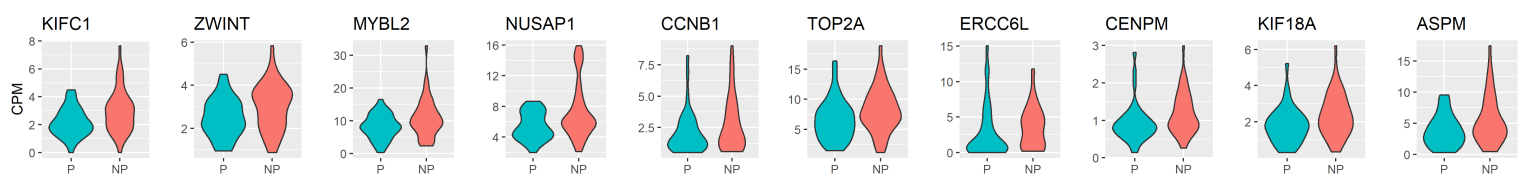

Figure 2. Violin plots comparing transcript abundance of GSEA-derived leading-edge genes in protected (P) and non-protected (NP) subjects pooled from four malaria vaccine trials. a Transcript abundance in counts per million (CPM) for the top ten leadingedge genes in DC.M3.2 Inflammation, the gene set showing the highest significant normalized enrichment score in the GSEA analysis (indicating significantly higher abundances in protected subjects). b Transcript abundance for the top ten leading-edge genes in LI.M4. 1 cell cycle (I), the gene set showing the lowest significant normalized enrichment score in the analysis (indicating significantly lower abundances in protected subjects).

\section{Correlates of protection shared across individual trials}

127 The analyses on the pooled set of preimmunization transcriptomes includes all samples aggregated across all four trials. To perform a more conservative analysis that limits correlates driven primarily by individual trials, we computed differential expression and performed GSEA for each trial separately, then identified genes and gene sets that consistently showed significant differences between protected and non-protected subjects across trials. As with the differential expression analysis on the pooled transcriptomes, the number of transcripts showing significant differences in abundance between protected and non-protected subjects was low in each trial ( $N=2$ for IMRAS, $N=0$ for BSPZV1 and MAL68, $N=14$ for CPS; FDR-adjusted $P$ value $<0.1$ ). To identify differences at the level of gene sets, we then performed the same type of GSEA as performed with the pooled samples. From the GSEA results on each trial, we then identified gene sets that showed significant positive enrichment scores consistently across all trials or consistently showed significant negative scores. Fourteen gene sets showed directionally-consistent, significant enrichment for genes with higher expression in protected subjects across trials, and two sets showed directionally-consistent, significant enrichment for genes with lower expression in protected subjects across trials (Fig. 3).

The 14 gene sets showing consistently higher transcript levels in protected subjects prior to immunization were predominantly associated with inflammatory responses, monocytes, and neutrophils. The two gene sets showing consistently lower transcript levels in protected subjects were associated with the cell cycle and protein synthesis. To determine the probability of seeing 16 gene sets significantly enriched across all four trials with consistent directionality in their 


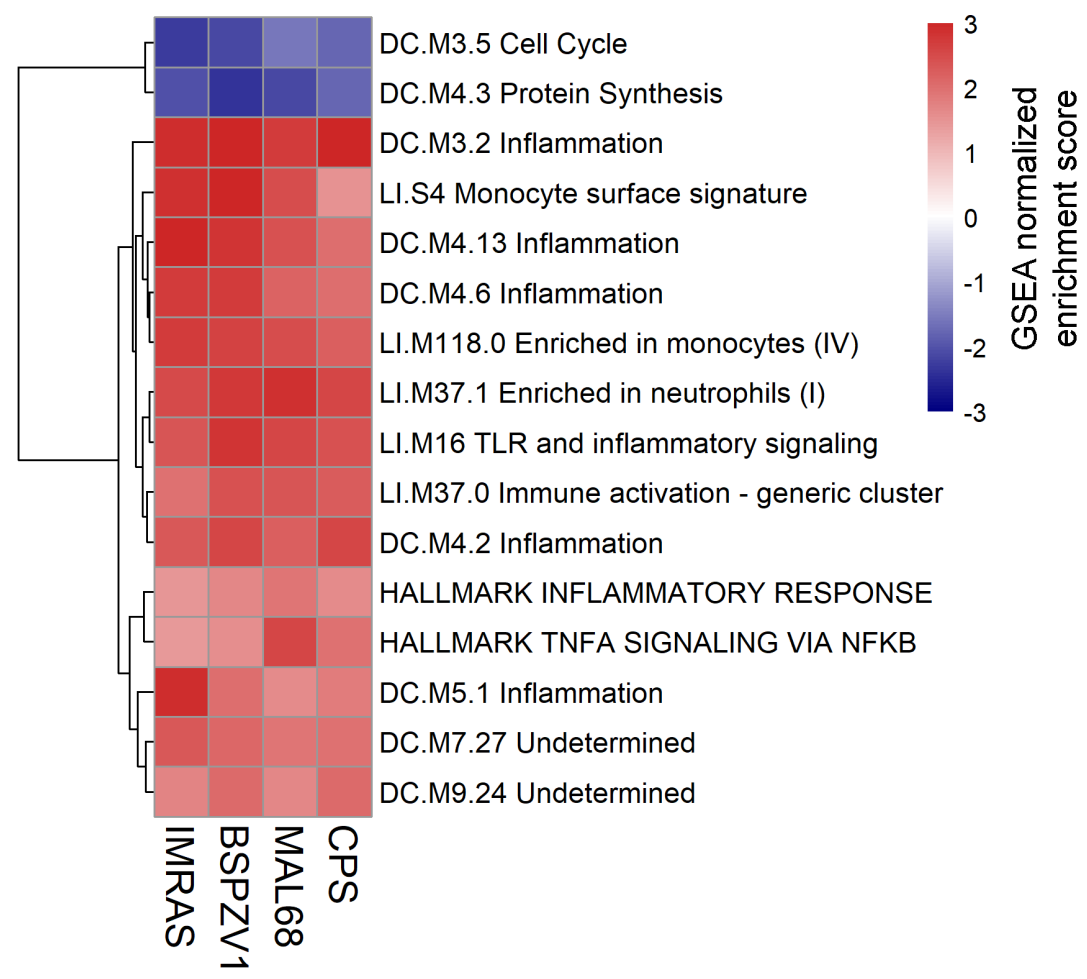

Figure 3. Heatmap of GSEA normalized enrichment scores for gene sets showing directionally-consistent, significant enrichment for gene transcripts with higher abundance in protected subjects across malaria vaccine trials or lower abundance across trials.

enrichment scores by chance, we randomly assigned protected/non-protected status to each trial subject, re-computed differential expression between protected and non-protected subjects, performed GSEA on the ranked DESeq2 results, and then recorded the number of gene sets showing directionally-consistent, significant enrichment in all four trials. This was done in a manner that preserved the original number of protected and non-protected subjects in each trial. We performed 10,000 iterations of this procedure, each of which used a unique set of protection assignments and found that the probability of seeing 16 or more directionally-consistent, significantly-enriched gene sets appear in all four trials was less than 0.007 .

While the gene sets used in our GSEA analysis are useful for characterizing gene groups according to their more general biological functions, they do not represent specific mechanistic molecular pathways. To identify such pathways that were consistently associated with protection at the preimmunization timepoint, we performed an Ingenuity Pathway Analysis (IPA $)^{31}$ on the set of common leading-edge genes collected from the gene sets in Fig. 3 that showed directionally-consistent, significant enrichment across vaccine trials. For each of these 16 functional gene sets, we collected the genes that appeared as GSEA leading-edge genes for that set in all four trials. Across these 16 gene sets, we found 98 such genes (Supplementary 
Table S1). Functionally profiling these genes collectively with IPA yielded significant enrichment (Fisher's exact test, FDR-adjusted $P$-value < 0.05) for 28 IPA Canonical Pathways (Fig. 4). Reflecting the GSEA results, the 28 pathways were primarily associated with inflammation, including many pathways in which Toll-like receptors (TLRs) and MYD88 participate. For each of the 98 genes input to IPA for this analysis, we computed the mean $\log _{2}$ fold-change differences in transcript abundance between protected and non-protected subjects across trials, and these values were used by IPA to identify which of the 28 Canonical Pathways showed a consistent activation or inactivation profile. The Canonical Pathway with the highest enrichment score that also showed a consistent activation or inactivation profile was the Toll-like Receptor Signaling pathway. Fig. 5 shows the IPA diagram for this pathway, highlighting the overlap between members of the 98-gene list and the pathway's molecular participants as well as the mean abundance differences of those overlapping participants when comparing protected to non-protected trial subjects.

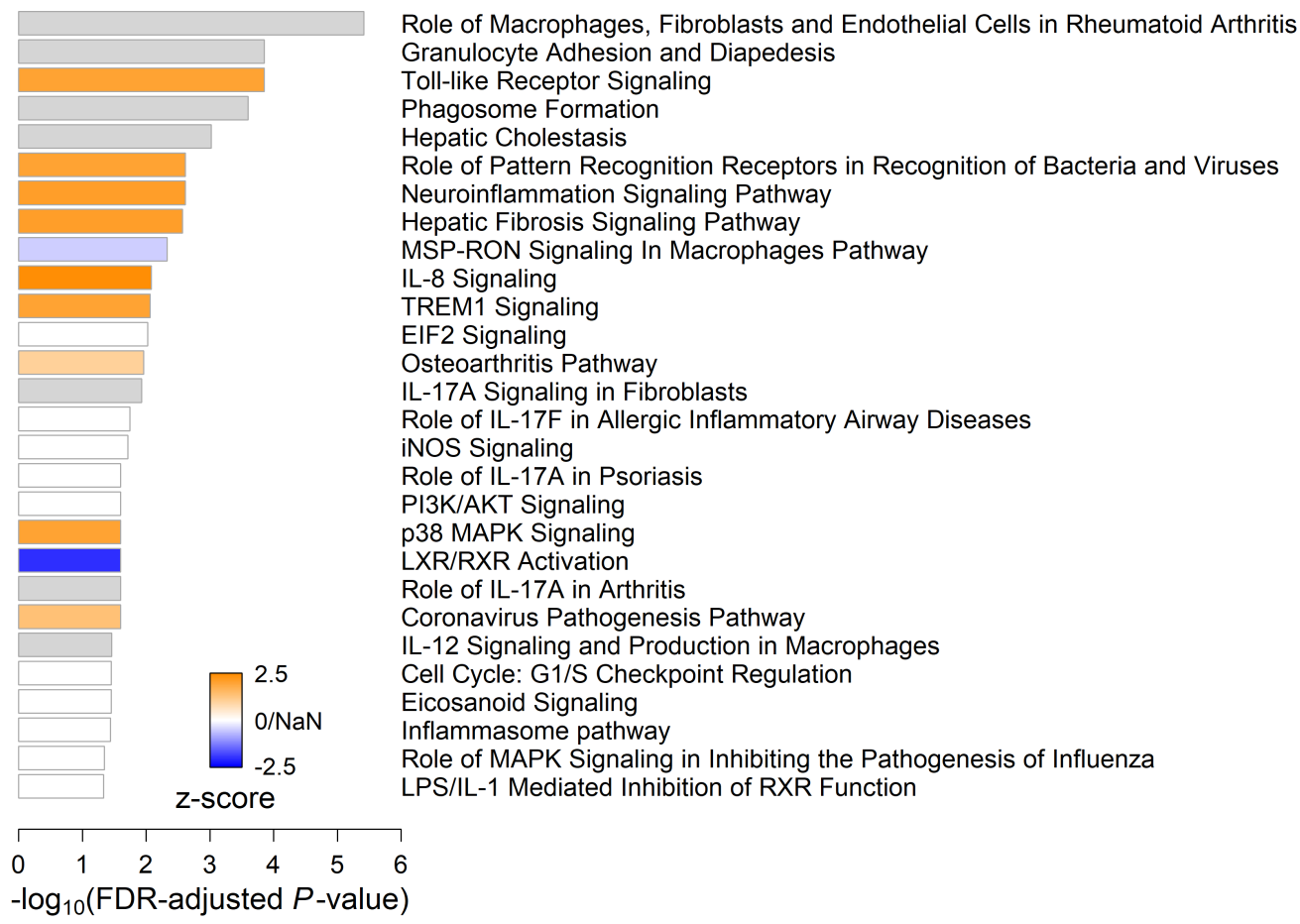

Figure 4. IPA Canonical Pathways analysis on common leading-edge genes from gene sets that showed directionally-consistent, significant GSEA enrichment scores across trials. Bar width indicates the strength of an IPA Canonical Pathway's enrichment for the leading-edge genes. Bar color indicates the IPA z-score, which is the degree to which the pathway is activated (orange) or inactivated (blue) based on mean $\log _{2}$ fold-changes in transcript abundance between protected and non-protected subjects for leading-edge genes found in the pathway. White bars indicate an ambiguous activation state $(z=0)$ or pathways ineligible for activation analysis because the overlap between common leading-edge genes and pathway participants used to compute the z-score was less than four (z-score $=\mathrm{NaN})$. Gray bars indicate that there is insufficient evidence in the IPA Knowledge Base to confidently predict an activation state for the pathway. 


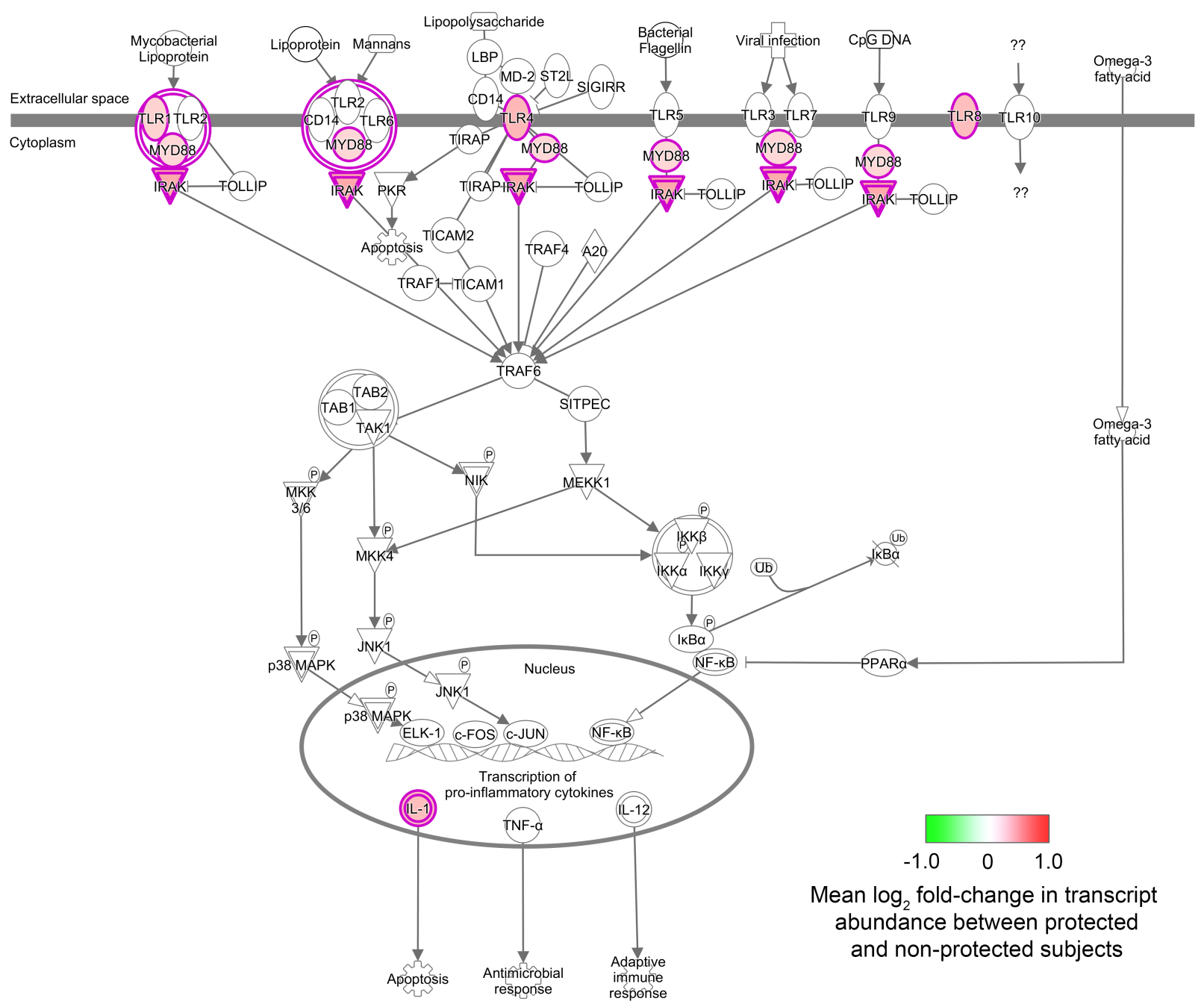

Figure 5. IPA network diagram of the Toll-like Receptor Signaling pathway, illustrating higher preimmunization activity in protected subjects. The pathway shows enrichment for six protection-associated leading-edge gene transcripts found across vaccine trials, indicated by icons with red borders. Icon fill color indicates the $\log _{2}$ fold-changes in transcript abundance when comparing protected to non-protected subjects, averaged across the four vaccine trials.

In addition to the Canonical Pathways analysis, we also performed an IPA Upstream Analysis, which provides hypotheses about which molecular perturbations might cause the expression differences observed in the 98-gene list. In the context of this study, this analysis can help identify pathway activators and inactivators (naturally-occurring or synthetic) with the potential to shift an individual's immunological state in the direction that is more consistent with a protective response to immunization. The molecules with high IPA activation z-scores $(\geq 2.0)$ showing significant FDR-adjusted $P$-values in this analysis included cytokines with proinflammatory activity (type I and type II interferons, IL1B, TNF, IL17A, IL33, IL18, IL6, IL12, IL1A, IL5), cytokine groups (IL12, IL1), lipopolysaccharides, colony stimulating factors (CSF2 and the 
medRxiv preprint doi: https://doi.org/10.1101/2021.08.13.21262031; this version posted September 5, 2021. The copyright holder for this preprint (which was not certified by peer review) is the author/funder, who has granted medRxiv a license to display the preprint in perpetuity.

All rights reserved. No reuse allowed without permission.

SPI1, TCF7L2, STAT3, RELA, IRF7), Toll-like receptors (TLR2, TLR4) as well as adapter proteins that participate in their signaling pathways (TICAM1, MYD88), other immunomodulators (tretinoin, ethanol, SPI1, TGM2, poly rl:rC-RNA, mycophenolic acid, POU2F2, KITLG), the protein synthesis regulator LARP1, thrombin, APP, signaling pathway enzymes and ligands (PARP1, PI3K, KITLG, the p38 MAPK group), the PDGF-BB complex, the growth hormone protein group, the C11 orf95-RELA gene fusion product, and ZBTB10. Based on the IPA knowledge base, increased activity of these molecules would shift expression of the 98 leadingedge genes in a manner consistent with their mean expression differences between protected and non-protected subjects across trials. The upstream molecules with low IPA activation zscores $(\leq-2.0)$ showing significance included the p38 MAPK inhibitor SB203580, the progesterone antagonist mifepristone, miR-155-5p, the alpha catenin protein group, the transcription factor MLXIPL, the transcription inhibitor actinomycin D, DIO3 and DUSP1, the gene fusion product ETV6-RUNX1, the immunosuppressant cyclosporin $A$, and the corticosteroid budesonide. Decreased activity of these molecules is predicted to induce expression changes in the 98 leading-edge genes consistent with those observed between protected and non-protected subjects. The full set of Upstream Analysis results are provided in Supplementary Table S2.

\section{Discriminatory power of protection-associated genes and gene sets}

The protection-associated genes and gene sets common across trials suggest there are transcriptomic features that might be used in classifiers that discriminate, on an individual basis, which vaccinees will mount a protective immune response to immunization and which will not. To assess the discriminatory power of these features, we developed and tested various scores based on transcript abundances (counts per million) of the 98 protection-associated, leadingedge genes described above as well as mean transcript levels corresponding to those genes within protection-associated gene sets. We also tested the discriminatory power of scores based on transcript abundance ratios between protection-associated genes and between gene sets. For all scoring strategies, individual scores were computed for each trial participant based on their preimmunization transcriptomic profile. We then assessed the discriminatory power of the score by varying the threshold used to classify individuals as protected or non-protected and then generating a receiver operating characteristic (ROC) curve from the sensitivity and specificity of the classifiers. We quantified the discriminatory power of each score based on the area under the curve (AUC) of the ROC results. We found that the ratio of mean leading-edge gene expression in the HALLMARK TNFA SIGNALING VIA NFKB gene set to that of the DC.M4.3 Protein synthesis gene set produced a score with the highest discriminatory power $(A \cup C=0.73,95 \%$ confidence interval 0.61-0.84, Fig. 6a). A Mann-Whitney $U$ test showed that this score was significantly different between the protected and non-protected subjects pooled from all four trials (FDR-adjusted $P$-value $=0.034$, Fig. 6b). The score incorporates expression information from eight members of the HALLMARK TNFA SIGNALING VIA NFKB gene set (BCL3, BTG1, CCRL2, CEBPD, CXCL1, PFKFB3, PTGS2, SLC2A3) and seven from the DC.M4.3 Protein synthesis set (RPL4, RPL5, RPL6, RPS3, RPS14, RPS18, TOMM7). 

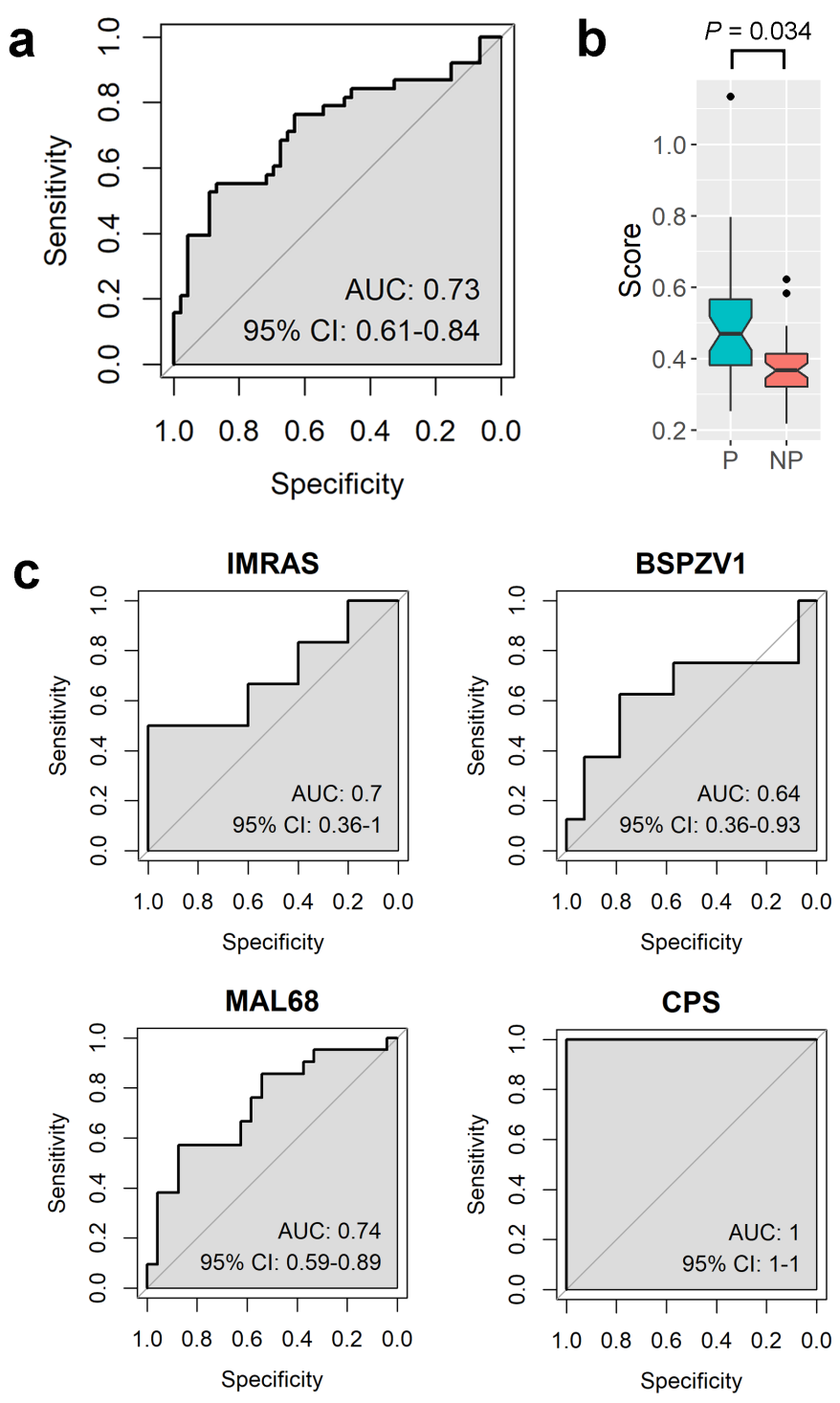

Figure 6. Discriminatory power of the best-performing preimmunization score for classifying protected and non-protected trial subjects. a Receiver operating characteristic $(R O C)$ curve showing performance of the score across 84 subjects pooled across trials. $\mathbf{b}$ Boxplots showing score distributions among subjects who were protected $(P)$ following malaria challenge and those who were not (NP). c ROC curves showing performance of the score within each individual trial. (AUC: area under the curve; $\mathrm{Cl}$ : confidence interval).

To test whether the discriminatory power of the best-performing score might be biased by specific trials in our study, we computed separate ROC curves for each trial using only their participating subjects and their corresponding scores. We found that the score showed comparable discriminatory power across trials (Fig. 6c). The score's performance was poorest in the BSPZV1 cohort (AUC=0.64, 95\% confidence interval 0.36-0.93) and highest in the CPS cohort where it perfectly discriminated between protected and non-protected subjects. 
medRxiv preprint doi: https://doi.org/10.1101/2021.08.13.21262031; this version posted September 5, 2021. The copyright holder for this preprint (which was not certified by peer review) is the author/funder, who has granted medRxiv a license to display the preprint in perpetuity.

All rights reserved. No reuse allowed without permission.

\section{Discussion}

237 Our findings indicate that a moderately elevated preimmunization inflammatory state, as revealed by RNA-seq transcriptomic measurements on whole blood or PBMCs, is associated with a protective response to malaria challenge following a variety of immunization strategies in adults. A heightened preimmunization inflammatory state has previously been linked to vaccineinduced protection against influenza in non-elderly adults ${ }^{6}$, and our results also link this finding to the malaria vaccination strategies analyzed here. Our findings contrast with studies investigating correlates of protection following hepatitis $B$ immunization where innate immune activation prior to immunization has been linked to non-protection in adults ${ }^{10-12}$.

Across the four trials we analyzed, transcripts from several inflammatory and innate response gene sets trended higher in protected subjects, and this trend was significant when treating gene sets as the unit of analysis. Analyses at the level of individual transcripts did not reveal significant differences between protected and non-protected subjects across trials. Thus, the expression differences we observed between these groups link protection to a moderately higher activation of inflammatory and innate response processes. While moderate, these differences are substantial enough to have potential utility in metrics that predict whether an individual subject will mount a protective vaccine response prior to immunization (Fig. 6). In future work, metrics based on the discriminatory transcriptomic features identified here might be combined with other protection-associated preimmunization measurements (e.g., cell type abundances) to create robust, accurate classifiers suitable for identifying individuals at risk for a poor vaccine response in a clinical setting.

The set of common leading-edge genes gathered from our GSEA results showed enrichment for more fine-grained signaling pathways, most of which included TLR4 as a participant. These results echo previous findings by Moncunill, et al. ${ }^{19}$ who identified TLR4 as a member of several gene signatures predictive of protection in RTS,S-vaccinated children. These signatures were derived from expression changes in preimmunization PBMCs that were stimulated with CSP and/or $P$. falciparum-infected erythrocytes. Their results suggested that higher preimmunization TLR4 expression may help the immune system respond more effectively to the RTS,S vaccine. As noted by these authors, the LPS-derived chemical monophosphoryl lipid A (MPL) used as an adjuvant in combination with the subunit RTS,S vaccine is a TLR4 agonist ${ }^{19,32,33}$. Our results, which link higher preimmunization expression of TLR1, TLR4 and TLR8 to protection, also suggest a role for TLR agonists as adjuvants in the development of a protective vaccine response. adults were immunized with fresh sporozoites delivered via mosquito bite under chloroquine prophylaxis. The best performing preimmunization signature derived from CSP-stimulated PBMCs for this trial linked protection with proinflammatory activity of participants in the canonical and non-canonical NFKB pathways. These pathway participants included CSF2 (also known as GM-CSF), and our IPA Upstream Analysis identified CSF2, RELA, and NFKB complex

278 activation as potential upstream perturbations contributing to the increased expression and 
medRxiv preprint doi: https://doi.org/10.1101/2021.08.13.21262031; this version posted September 5, 2021. The copyright holder for this preprint (which was not certified by peer review) is the author/funder, who has granted medRxiv a license to display the preprint in perpetuity.

All rights reserved. No reuse allowed without permission.

transcript abundance differences we observed between protected and non-protected subjects. Also included in the CPS signature is ITGA2, an integrin that contributes to p38 MAPK signaling which in turn controls the activity of the RELA NFKB subunit. The p38 MAPK signaling pathway was also enriched for leading-edge genes associated with protection across trials in our IPA Canonical Pathways analysis (Fig. 4). The leading-edge genes participating in this pathway included CREB5, IL18RAP, IL1RN and IRAK3, all of which showed elevated expression in protected subjects across trials. These results suggest that, although we did not see overlap between our set of 98 leading-edge genes and the genes in the preimmunization signature associated with CSP-stimulation in the chloroquine prophylaxis trial analyzed by Moncunill, et al., NFKB-mediated inflammation pathways appear to be a critical determinant of protection in both studies.

An important difference between the Moncunill, et al. study and ours is that our transcriptomic measurements were taken from unstimulated tissues and therefore present snapshots of subjects' preimmunization immunological states whereas the Moncunill, et al. measurements reflect immunological responses to antigen stimulation. Taken together, these results associate protection with an innate immune system that is simultaneously more activated prior to immunization and more capable of responding to immunization. These results may be driven by higher-than-average innate immune functionality in protected subjects or lower-than-average functionality in non-protected subjects, or a combination of both. A recent report by Yap, et al. ${ }^{21}$ provides evidence suggesting preimmunization correlates of protection may be driven by actively decreased inflammatory functions in non-protected subjects. The authors report that higher baseline expression of T cell inhibitory ligands CTLA-4 and TIM-3 in CD4 ${ }^{+}$T cells is associated with a "slow-responder" phenotype in adults that is less likely to result in sterile protection following immunization via controlled human malaria infection under chloroquine prophylaxis (CHMI-CPS). Increases in these inhibitory ligands can result from mechanisms that protect against inflammation-induced damage (for example in response to chronic parasitemia in malaria-endemic areas). The authors argue that these inhibitory ligands may interfere with NFKB signaling, which is preferentially activated in subjects who mount a protective response to this type of immunization, and this leads to insufficient production of IFN- $\gamma$. Rapid induction of IFN- $\gamma$ following immunization is a characteristic of a "fast-responder" phenotype in CHMI-CPS trials that is more likely to result in sterile protection. Consistent with these findings, our IPA Upstream Analysis identified increased IFN- $\gamma$ as a perturbation that would generate expression changes consistent with those observed between protected and non-protected individuals. IFN- $\gamma$ had the highest z-score in this analysis, indicating strong agreement between a hypothetical increase in IFN- $\gamma$ and our observed expression patterns showing higher inflammation in protected subjects prior to immunization. Given this consistency between our findings and those of Yap, et al., it may be that non-protected individuals in our study had a less successful immunization response due to protective inflammatory inhibition. However, the group of individuals we analyzed was heterogeneous; therefore, we cannot rule out attributing protection to higher-than-average innate responses among protected subjects. Furthermore, these two interpretations are not mutually exclusive: our results may simultaneously reflect immunosuppressive factors in non-protected subjects and immuno-activating factors in protected subjects. 
medRxiv preprint doi: https://doi.org/10.1101/2021.08.13.21262031; this version posted September 5, 2021. The copyright holder for this preprint (which was not certified by peer review) is the author/funder, who has granted medRxiv a license to display the preprint in perpetuity.

All rights reserved. No reuse allowed without permission.

The results presented here suggest that assessing malaria vaccinee's preimmunization status may lead to important correlates of protection and tailoring immunization regimes accordingly may emerge as an important element to ensuring a protective response to immunization. Moreover, our results also offer potential pharmacological interventions that may coax an individual's immune state into one that is primed to respond effectively to immunization.

\section{Methods}

\section{Transcriptomic data sources}

Transcriptomic data were aggregated from adult participants in four malaria vaccine trials that included RNA-seq measurements at preimmunization timepoints and an assessment of protection against infectious $P$. falciparum following immunization. The trials, described below, are summarized in Table 1. Each trial investigated a different malaria vaccine candidate and, together, represent cohorts from malaria-naïve and non-naïve populations. From these trials, 85 preimmunization transcriptomic profiles were available to investigate correlates of protection.

\section{Trial 1: IMRAS}

The IMRAS trial22 (ClinicalTrials.gov identifier NCT01994525: https://clinicaltrials.gov/ct2/show/NCT01994525) was performed in malaria-naïve participants recruited from the United States. The trial assessed the safety, tolerability, and biomarkers of protection of immunization via radiation-attenuated sporozoites delivered by infectious mosquito bites. Preimmunization transcriptomics data were obtained from whole blood samples collected on the day of the first immunization from 11 immunized participants in the trial's Cohort 1, each of whom received five doses of radiation-attenuated $P$. falciparum via mosquito bite. Three weeks after the fifth immunization, participants were challenged via $P$. falciparum-infected mosquito bites. To facilitate the identification of correlates of protection, the IMRAS immunization regimen was designed to achieve approximately $50 \%$ sterile protection in immunized participants: 6 out of the 11 immunized participants (54\%) in Cohort 1 were protected following challenge.

\section{Trial 2: BSPZV1}

The BSPZV1 trial ${ }^{23}$ (ClinicalTrials.gov identifier NCT02132299: https://clinicaltrials.gov/ct2/show/NCT02132299) was performed in male, malaria-exposed recruits from Tanzania. The trial was designed to determine if protective immune responses against $P$. falciparum infection could be generated in a relatively non-immune population within a malaria-endemic country through immunization with aseptic, cryopreserved, live, radiationattenuated $P$. falciparum sporozoites delivered intravenously (IV). Preimmunization transcriptomic data from 22 immunized participants were obtained from whole blood samples collected on the day of the first immunization. Each participant received five immunizations and, 
medRxiv preprint doi: https://doi.org/10.1101/2021.08.13.21262031; this version posted September 5, 2021. The copyright holder for this preprint (which was not certified by peer review) is the author/funder, who has granted medRxiv a license to display the preprint in perpetuity.

All rights reserved. No reuse allowed without permission.

three weeks after the last, were challenged with infectious, aseptic, cryopreserved $P$. falciparum sporozoites delivered IV.

\section{Trial 3: MAL68}

The MAL68 trial $^{24}$ (ClinicalTrials.gov identifier NCT01366534: https://clinicaltrials.gov/ct2/show/NCT01366534) compared the ability of two malaria vaccination regimens to elicit protection against malaria challenge in malaria-naïve recruits from the United States. The first regimen consisted of three doses of the RTS,S/AS01 vaccine, which combines a recombinant protein encoding part of the malaria parasite's circumsporozoite (CSP) protein, the hepatitis B surface antigen, and the AS01 adjuvant. This adjuvant includes the Toll-like receptor 4 (TLR4) agonist 3-O-desacyl-4'-monophosphoryl lipid A (MPL) and the QS-21 saponin $^{32}$. The second regimen was the same as the first except a different vaccine, Ad35.CS.01, was used for the first immunization. Ad35.CS.01 consists of replication-deficient adenovirus 35 expressing recombinant CSP. Preimmunization transcriptomics from 45 immunized participants (21 from the first regimen, 24 from the second) were obtained from PBMCs collected on the day of the first immunization. Participants were challenged with $P$. falciparum-infected mosquitos two weeks following the third immunization.

\section{Trial 4: CPS}

The CPS trial|25,26 (ClinicalTrials.gov identifier NCT01218893: https://clinicaltrials.gov/ct2/show/NCT01218893) investigated the influence of dosing level on the development of protection against malaria challenge when immunizing malaria-naïve participants using $P$. falciparum-infected mosquito bites obtained under chloroquine prophylaxis. Participants were recruited in The Netherlands and received three immunizations separated by 4-week intervals. They were challenged 19 weeks after the last immunization by exposure to bites from five mosquitoes infected with a homologous $P$. falciparum strain. Preimmunization transcriptomics were obtained from whole blood samples collected one week prior to the first immunization and were from seven members of the study group immunized with 15 mosquito bites total.

\section{Transcriptomic analyses}

Raw RNA-seq sequencing data were aligned to the hg19 human reference genome using a previously described pipeline ${ }^{34}$. Briefly, read pairs were adjusted to set base calls with phred scores less than 5 to "N", and read pairs for which either end had less than 30 unambiguous base calls were removed. The latter step indirectly removes pairs containing mostly adaptor sequences. Read pairs were then aligned to the genome using STAR ${ }^{35}$ version 2.3.1 and gene counts were computed using HTSeq ${ }^{36}$ version 0.6.0.

Differential expression analyses comparing preimmunization sample counts from challengeprotected trial subjects to non-protected subjects were performed using DESeq2 ${ }^{37}$ version 1.28.0. Principal Component Analysis (PCA) was used to identify outliers among transcriptomic samples: If a sample's first or second principal component value was more than three standard 
deviations from the corresponding principal component's mean, they were excluded from downstream analyses. One sample from the CPS trial was identified as an outlier based on PCA. To account for batch effects among trials, we used a DESeq2 design formula that included a trial term as well as a protected/non-protected classification term. Differential expression analyses performed on samples from the same trial only included the latter term.

Gene Set Enrichment Analysis (GSEA) ${ }^{27}$ was performed on gene lists ranked by the DESeq2 Wald test statistic using the fgsea package ${ }^{38}$ version 1.14.0 in R. Gene sets used for GSEA were obtained through the tmod R package ${ }^{39}$ and the MSigDB Hallmark gene set $^{30}$ download site (http://www.gsea-msigdb.org/gsea/msigdb/genesets.jsp?collection=H). Ingenuity Pathway Analysis (IPA) content version 62089861 was used to generate all IPA-based results. Receiver operating characteristic analyses were performed using the $\mathrm{pROC}$ package ${ }^{40}$ in $\mathrm{R}$. For all statistical tests, FDR-adjusted $P$-values (Benjamini-Hochberg method ${ }^{41}$ ) less than 0.05 were considered significant unless otherwise indicated.

\section{Data Availability}

413 Raw sequencing data for the MAL68 trial is publicly available through Sequence Read Archive 414 BioProject PRJNA401870. Raw sequencing data for the CPS trial is publicly available at 415 Sequence Read Archive BioProject PRJNA381264. Data for the IMRAS and BSPZV1 trials will 416 be available through the ImmPort portal (immport.org).

\section{Acknowledgements}

418 We would like to thank Nana Minkah for helpful discussions regarding preimmunization 419 correlates, and all volunteers who participated in the clinical trials that were analyzed.

\section{Author contributions}

421 M.L.N. performed all analyses, interpreted findings, and prepared the manuscript. F.J.D.

422 identified and obtained transcriptomic datasets for analysis, generated counts from sequencing 423 data, helped guide analyses, and edited the manuscript. Y.D. generated counts from

424 sequencing data and edited the manuscript. J.D.A. and K.D.S. conceived the study, interpreted 425 findings, and edited the manuscript.

\section{Competing Interests}

427 The authors declare no competing interests. 
medRxiv preprint doi: https://doi.org/10.1101/2021.08.13.21262031; this version posted September 5, 2021. The copyright holder for this preprint (which was not certified by peer review) is the author/funder, who has granted medRxiv a license to display the preprint in perpetuity.

All rights reserved. No reuse allowed without permission.

\section{Funding Statement}

429 This work was supported by National Institutes of Health grants U19Al128914 (to K.D.S.) and 430 P41GM109824 (to J.D.A.) as well as Bill \& Melinda Gates Foundation grant GHVAP NG-ID18431 Stuart. 


\section{References}

433 1. Petra, Z. \& Nigel, C. Factors that influence the immune response to vaccination. Clin. Microbiol.

434 Rev. 32, e00084-18 (2019).

435 2. Duffy, P. E. \& Patrick Gorres, J. Malaria vaccines since 2000: progress, priorities, products. npj

$436 \quad$ Vaccines 5, 48 (2020).

437 3. RTS,S Clinical Trials Partnership. Efficacy and safety of RTS,S/AS01 malaria vaccine with or without a booster dose in infants and children in Africa: final results of a phase 3 , individually randomised, controlled trial. Lancet 386, 31-45 (2015).

4. Mwakingwe-Omari, A. et al. Two chemoattenuated PfSPZ malaria vaccines induce sterile hepatic immunity. Nature 595, 289-294 (2021).

5. Datoo, M. S. et al. Efficacy of a low-dose candidate malaria vaccine, R21 in adjuvant Matrix-M, with seasonal administration to children in Burkina Faso: a randomised controlled trial. Lancet 397, 1809-1818 (2021).

6. HIPC-CHI Signatures Project Team \& HIPC-I Consortium. Multicohort analysis reveals baseline transcriptional predictors of influenza vaccination responses. Sci. Immunol. 2, eaal4656 (2017).

7. Tsang, J. S. et al. Global analyses of human immune variation reveal baseline predictors of postvaccination responses. Cell 157, 499-513 (2014).

8. Frasca, D. et al. Unique biomarkers for B-cell function predict the serum response to pandemic H1N1 influenza vaccine. Int. Immunol. 24, 175-182 (2012).

9. Kennedy, R. B. et al. The composition of immune cells serves as a predictor of adaptive immunity in a cohort of 50- to 74-year-old adults. Immunology 148, 266-275 (2016).

10. Fourati, S. et al. Pre-vaccination inflammation and B-cell signalling predict age-related hyporesponse to hepatitis B vaccination. Nat. Commun. 7, 10369 (2016).

11. Bartholomeus, E. et al. Transcriptome profiling in blood before and after hepatitis B vaccination shows significant differences in gene expression between responders and non-responders. Vaccine 36, 6282-6289 (2018).

12. Qiu, S. et al. Significant transcriptome and cytokine changes in hepatitis B vaccine nonresponders revealed by genome-wide comparative analysis. Hum. Vaccin. Immunother. 14, 17631772 (2018).

13. Kotliarov, Y. et al. Broad immune activation underlies shared set point signatures for vaccine responsiveness in healthy individuals and disease activity in patients with lupus. Nat. Med. 26, 618-629 (2020).

14. Ubillos, I. et al. Baseline exposure, antibody subclass, and hepatitis B response differentially affect malaria protective immunity following RTS,S/AS01E vaccination in African children. BMC Med. 16, 197 (2018).

15. Stanisic, D. I. \& McCall, M. B. B. Correlates of malaria vaccine efficacy. Expert Rev. Vaccines 20, 143-161 (2021).

16. Warimwe, G. M. et al. Peripheral blood monocyte-to-lymphocyte ratio at study enrollment predicts efficacy of the RTS,S malaria vaccine: analysis of pooled phase II clinical trial data. BMC Med. 11, 184 (2013).

17. Ishizuka, A. S. et al. Protection against malaria at 1 year and immune correlates following PfSPZ vaccination. Nat. Med. 22, 614-623 (2016).

18. Sissoko, M. S. et al. Safety and efficacy of PfSPZ Vaccine against Plasmodium falciparum via direct venous inoculation in healthy malaria-exposed adults in Mali: a randomised, double-blind phase 1 trial. Lancet Infect. Dis. 17, 498-509 (2017).

19. Moncunill, G. et al. Antigen-stimulated PBMC transcriptional protective signatures for malaria 
immunization. Sci. Transl. Med. 12, eaay8924 (2020).

20. Nielsen, C. M. et al. RTS,S malaria vaccine efficacy and immunogenicity during Plasmodium falciparum challenge is associated with HLA genotype. Vaccine 36, 1637-1642 (2018).

21. Yap, X. Z., McCall, M. B. B. \& Sauerwein, R. W. Fast and fierce versus slow and smooth: Heterogeneity in immune responses to Plasmodium in the controlled human malaria infection model. Immunol. Rev. 293, 253-269 (2020).

22. Hickey, B. et al. IMRAS-A clinical trial of mosquito-bite immunization with live, radiationattenuated $P$. falciparum sporozoites: Impact of immunization parameters on protective efficacy and generation of a repository of immunologic reagents. PLoS One 15, e0233840 (2020).

23. Jongo, S. A. et al. Safety, immunogenicity, and protective efficacy against controlled human malaria infection of Plasmodium falciparum sporozoite vaccine in Tanzanian adults. Am. J. Trop. Med. Hyg. 99, 338-349 (2018).

24. Ockenhouse, C. F. et al. Ad35.CS.01 - RTS,S/AS01 heterologous prime boost vaccine efficacy against sporozoite challenge in healthy malaria-naïve adults. PLoS One 10, e0131571 (2015).

25. Bijker, E. M. et al. Cytotoxic markers associate with protection against malaria in human volunteers immunized with Plasmodium falciparum sporozoites. J. Infect. Dis. 210, 1605-1615 (2014).

26. Tran, T. M. et al. Whole-blood transcriptomic signatures induced during immunization by chloroquine prophylaxis and Plasmodium falciparum sporozoites. Sci. Rep. 9, 8386 (2019).

27. Subramanian, A. et al. Gene set enrichment analysis: A knowledge-based approach for interpreting genome-wide expression profiles. Proc. Natl. Acad. Sci. 102, 15545-15550 (2005).

28. Chaussabel, D. et al. A modular analysis framework for blood genomics studies: Application to systemic lupus erythematosus. Immunity 29, 150-164 (2008).

29. Li, S. et al. Molecular signatures of antibody responses derived from a systems biological study of 5 human vaccines. Nat. Immunol. 15, 195-204 (2014).

30. Liberzon, A. et al. The Molecular Signatures Database Hallmark gene set collection. Cell Syst. 1, 417-425 (2015).

31. Krämer, A., Green, J., Pollard Jr, J. \& Tugendreich, S. Causal analysis approaches in Ingenuity Pathway Analysis. Bioinformatics 30, 523-530 (2014).

32. Kazmin, D. et al. Systems analysis of protective immune responses to RTS,S malaria vaccination in humans. Proc. Natl. Acad. Sci. 114, 2425-2430 (2017).

33. Laurens, M. B. RTS,S/AS01 vaccine (Mosquirix ${ }^{\mathrm{TM}}$ ): an overview. Hum. Vaccin. Immunother. 16, 480-489 (2020).

34. Thompson, E. G. et al. Host blood RNA signatures predict the outcome of tuberculosis treatment. Tuberculosis (Edinb). 107, 48-58 (2017).

35. Dobin, A. et al. STAR: Ultrafast universal RNA-seq aligner. Bioinformatics 29, 15-21 (2013).

36. Anders, S., Pyl, P. T. \& Huber, W. HTSeq-a Python framework to work with high-throughput sequencing data. Bioinformatics 31, 166-169 (2015).

37. Love, M. I., Huber, W. \& Anders, S. Moderated estimation of fold change and dispersion for RNAseq data with DESeq2. Genome Biol. 15, 550 (2014).

38. Korotkevich, G. et al. Fast gene set enrichment analysis. bioRxiv 60012 (2021).

39. Weiner 3rd, J. \& Domaszewska, T. tmod: an R package for general and multivariate enrichment analysis. PeerJ Prepr. 4, e2420v1 (2016).

40. Robin, X. et al. pROC: an open-source package for R and S+ to analyze and compare ROC curves. BMC Bioinformatics 12, 77 (2011).

41. Benjamini, Y. \& Hochberg, Y. Controlling the false discovery rate: A practical and powerful approach to multiple testing. J. R. Stat. Soc. Ser. B 57, 289-300 (1995). 